\title{
Medical Image of the Month: Ludwig's Angina
}

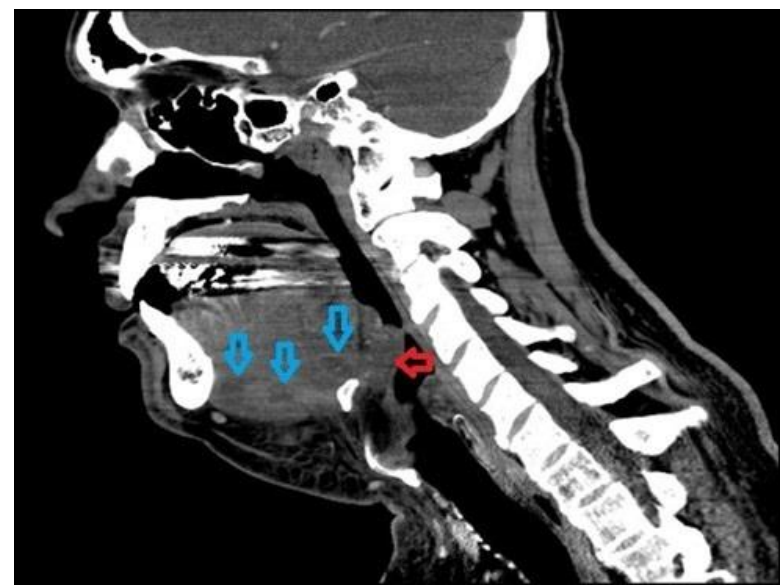

Figure 1. A coronal reconstruction of the patient's initial post-contrast maxillofacial CT demonstrates swelling of the soft tissues of the floor of the mouth consistent with phlegmonous changes and early abscess formation (blue arrows). There is also swelling in the region of the vallecula and epiglottis (red arrow).

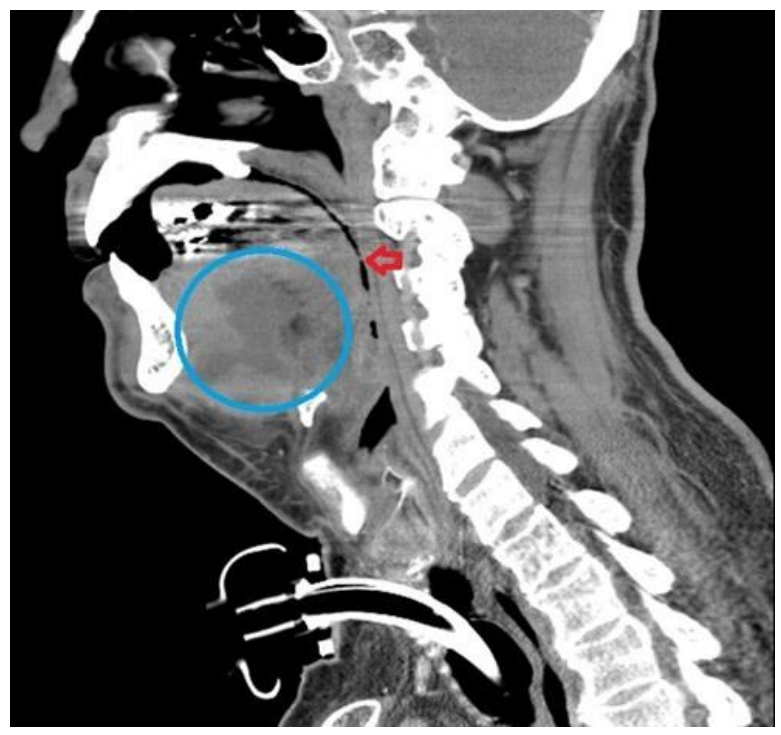

Figure 2. A coronal reconstruction of the patient's follow-up post-contrast maxillofacial CT after placement of a tracheostomy tube demonstrates marked progression of the swelling of the soft tissues of the floor of the mouth with development of a large, illdefined abscess in the floor of the mouth (blue circle). Note the marked, progressive narrowing of the oropharynx (red arrow) over a period of approximately 24 hours.

Case Presentation: A 65-year-old gentleman with a history of insulin-dependent diabetes mellitus presented to the emergency room with a chief complaint of two days of difficulty swallowing and jaw pain. Four days prior to presentation, he had extensive dental work performed to address multiple dental caries. On arrival to the emergency room, he was noted to be tachycardic with difficulty swallowing his saliva and liquids. On physical examination, he had difficulty opening his mouth with marked swelling of 
his tongue. He also had marked swelling of the soft tissues of the floor of the mouth with palpable adenopathy. A maxillofacial CT with contrast (Figure 1) was performed which demonstrated extensive edema and early abscess formation in the floor of the mouth. He was initially admitted to the general medicine floor and started on broadspectrum antibiotics. Over the course of the next 12 hours, he began to have increased difficulty breathing and was unable to swallow his own secretions. He was promptly transferred to the ICU where a fiberoptic nasotracheal intubation was attempted at bedside but was unable to be performed given the extensive soft tissue swelling in the posterior oropharynx. An emergent awake tracheostomy was subsequently performed by ENT. A repeat maxillofacial CT with contrast (Figure 2) demonstrated marked progression of the inflammatory changes and abscess formation in the floor of the mouth consistent with progressive Ludwig's angina. The combination of prompt surgical drainage and broad-spectrum antibiotics resulted in marked clinical improvement over the next 72 hours. The patient's final tissue cultures grew Streptococcus viridans.

Ludwig's angina is a potentially life-threatening gangrenous cellulitis of the neck and floor of the mouth which is characterized by progressive submandibular swelling with elevation and posterior displacement of the tongue. Odontogenic infections are the cause for most cases. Pre-existing medical conditions which predispose patients to the development of Ludwig's angina include diabetes mellitus, malnutrition, alcoholism, and immunocompromised states (i.e. AIDS and organ transplantation).

In the early stages of the disease, patients may be managed with observation and intravenous antibiotics to cover for $\beta$-hemolytic streptococcus and anaerobic organisms. The most life-threatening complication of Ludwig's angina is airway obstruction. Immediate involvement of an anesthesiologist and ENT are crucial in the management of this condition. Blind nasotracheal intubation should not be attempted in these patients given the potential for bleeding and abscess rupture. Flexible nasotracheal intubation requires skill and experience. If flexible nasotracheal intubation is not possible, a cricothyrotomy and tracheostomy under local anesthesia can be performed in the emergent setting. An elective awake tracheostomy is a safer and more logical method of airway management in patients with fully developed Ludwig's angina.

Lauren Estep, MD and Tammer El-Aini, MD Department of Pulmonary, Critical Care, Allergy and Sleep University of Arizona College of Medicine Tucson, AZ USA

\section{References}

1. Hasan W, Leonard D, Russell J. Ludwig's Angina-A Controversial Surgical Emergency: How We Do It. Int J Otolaryngol. 2011;2011:231816. [CrossRef] [PubMed]

2. Candamourty R, Venkatachalam S, Babu MR, Kumar GS. Ludwig's Angina - An emergency: A case report with literature review. J Nat Sci Biol Med. 2012 Jul;3(2):206-8. [CrossRef] [PubMed] 